\title{
A NOTE ON LINEAR SYSTEMS ON $K$-3 SURFACES
}

\author{
ALLEN TANNENBAUM
}

\begin{abstract}
A simple necessary and sufficient condition is given for a general member of the complete linear system $|Y|$ to be irreducible and nonsingular where $Y$ is a reduced, connected curve on a $K-3$ surface.
\end{abstract}

1. Introduction. It is well known that for $F$ a nonsingular rational projective irreducible surface (all our schemes will be defined over a fixed algebraically closed field of characteristic 0), given a reduced connected curve $Y$ on $F$ all of whose irreducible components have strictly negative intersection number with a canonical divisor $K_{F}$, a general member of the complete linear system $|Y|$ is nonsingular and irreducible.

In this note we will prove the following analogous theorem for linear systems on $K-3$ surfaces.

THEOREM. Let $F$ be a $K-3$ surface (i.e. a nonsingular projective irreducible surface with $H^{1}\left(F, \vartheta_{F}\right)=0$ and $\left.\vartheta_{F} \cong \vartheta_{F}\left(K_{F}\right)\right)$, and let $Y$ be a reduced, connected curve on $F$. Then a general member of $|Y|$ is nonsingular and irreducible if and only if for every expression $Y=Y_{1}^{\prime}+\cdots+Y_{q}^{\prime}$ of $Y$ as a sum of connected curves $(q \geqslant 2), \Sigma_{i<j} Y_{i}^{\prime} \cdot Y_{j}^{\prime}$ $\geqslant q$.

The author would like to thank the referee for suggesting the proof of Lemma (2.1) below for the case $C^{2}=0$.

2. The proof of the theorem. We begin with some well-known facts about curves on a $K-3$ surface $F$. First note that since $\theta_{F}\left(K_{F}\right) \cong \vartheta_{F}$, from the adjunction formula, $C^{2}=2 p_{a}(C)-2$ for $C \subset F$ a curve. Moreover if $C$ is a reduced connected curve, then by Riemann-Roch $\operatorname{dim}|C|=p_{a}(C)$. Indeed we have that

$$
\begin{aligned}
h^{0}\left(\vartheta_{F}(C)\right)-h^{1}\left(\vartheta_{F}(C)\right)+h^{2}\left(\Theta_{F}(C)\right) & =C \cdot\left(C-K_{F}\right) / 2+1+h^{2}\left(\vartheta_{F}\right) \\
& =p_{a}(C)+h^{2}\left(\Theta_{F}\right) \\
& =p_{a}(C)+1 .
\end{aligned}
$$

By Serre duality $h^{2}\left(\vartheta_{F}(C)\right)=h^{0}\left(\vartheta_{F}(-C)\right)=0$, and so we must show that $h^{1}\left(\vartheta_{F}(C)\right)$ $=0$ or equivalently (again by Serre duality) that $h^{1}\left(\theta_{F}(-C)\right)=0$. Note however since $C$ is reduced, connected $H^{0}\left(\theta_{C}\right) \cong H^{0}\left(\theta_{F}\right) \cong k$, and therefore applying the long exact cohomology sequence to the exact sequence $0 \rightarrow \theta_{F}(-C) \rightarrow \theta_{F} \rightarrow \theta_{C} \rightarrow 0$ and using the fact that $h^{1}\left(\theta_{F}\right)=0$, we get the desired conclusion.

Received by the editors November 4, 1981.

1980 Mathematics Subject Classification. Primary 14J99; Secondary 14C99.

(1) 1982 American Mathematical Society 0002-9939/82/0000-0773/\$01.75 
We can now state the following lemma.

LEMMA (2.1). Let $C$ be an arbitrary integral curve on a $K-3$ surface $F$. Then a general member of $|C|$ is nonsingular.

Proof. If $C^{2}<0$, then it is trivial to show that $C$ is nonsingular, rational. If $C^{2}>0$, then by $[2,(3.1)$, p. 611$]$, we have that $|C|$ is base point free, and hence we get the required conclusion from Bertini's theorem (recall that we are working over an algebraically closed field of characteristic 0 ). So we are left with the case $C^{2}=0$. But here we have that $\operatorname{dim}|C|=p_{a}(C)=1>0$ so that two general elements of $|C|$ intersect properly. Since $C^{2}=0$ they are disjoint, so again by Bertini a general element of $|C|$ is nonsingular. Q.E.D.

Proof of The Theorem (2.2). By (2.1) it is enough to show that for $Y$ as in the theorem a general member of $|Y|$ is irreducible if and only if for every expression $Y=Y_{1}^{\prime}+\cdots+Y_{q}^{\prime}$ of $Y$ as a sum of connected curves $(q \geqslant 2), \Sigma_{i<j} Y_{i}^{\prime} \cdot Y_{j}^{\prime} \geqslant q$. Suppose first that a general member of $|Y|$ is irreducible. Let $Y=Y_{1}^{\prime}+\cdots+Y_{q}^{\prime}$ be an expression of $Y$ as a sum of connected curves. Then

$$
\begin{aligned}
\operatorname{dim}|Y| & =p_{a}(Y)=p_{a}\left(Y_{1}^{\prime}+\cdots+Y_{q}^{\prime}\right) \\
& =\sum_{i=1}^{q} p_{a}\left(Y_{i}^{\prime}\right)+\sum_{i<j} Y_{i}^{\prime} \cdot Y_{j}^{\prime}-q+1 \\
& =\sum_{i=1}^{q} \operatorname{dim}\left|Y_{i}^{\prime}\right|+\sum_{i<j} Y_{i}^{\prime} \cdot Y_{j}^{\prime}-q+1 .
\end{aligned}
$$

Clearly since a general member of $|Y|$ is irreducible, we must have $\operatorname{dim}|Y|>$ $\sum_{i=1}^{q} \operatorname{dim}\left|Y_{i}^{\prime}\right|$ so that $\sum_{i<j} Y_{i}^{\prime} \cdot Y_{j}^{\prime} \geqslant q$.

Conversely suppose that a general member $Z \in|Y|$ is reducible, say $Z=Z_{1}$ $+\cdots+Z_{q}$ with the $Z_{i}$ irreducible, $i=1, \ldots, q$. (Note that since $Y$ is reduced, $Z$ is reduced, and hence $Z_{i} \neq Z_{j}$ for $i \neq j$.) Then there exist $Y_{i}^{\prime}$ connected sums of irreducible components of $Y$ such that $Z_{i}$ is a generalization of $Y_{i}^{\prime}$ in the linear system $\left|Z_{i}\right|=\left|Y_{i}^{\prime}\right|$ for each $i=1, \ldots, q$. But then certainly

$$
\operatorname{dim}|Y|=\sum_{i=1}^{q} \operatorname{dim}\left|Z_{i}\right|=\sum_{i=1}^{q} \operatorname{dim}\left|Y_{i}^{\prime}\right| .
$$

On the other hand from (+) above, this would imply that $\Sigma_{i<j} Y_{i}^{\prime} \cdot Y_{j}^{\prime}=q-1<q$ completing the proof of the theorem. Q.E.D.

EXAMPLE (2.3). In case $Y=Y_{1}+Y_{2}$ has two irreducible components, the theorem means that a general member of $|Y|$ is irreducible if and only if $Y_{1} \cdot Y_{2} \geqslant 2$. We give here an example of a curve $Y=Y_{1}+Y_{2}$ on a $K-3$ surface $F$ such that $Y_{1} \cdot Y_{2}=1$, and therefore is not a degeneration of a nonsingular irreducible curve on $F$.

Indeed, let $F$ be a nonsingular quartic surface in $\mathbf{P}^{3}$ which contains a line $l$ ( $F$ is well known to be a $K-3$ surface). Let $H$ be a general hyperplane section on $F$, so that $H$ is isomorphic to a nonsingular plane quartic curve. Then setting $Y=H+l$, we get that $H \cdot l=1$, and so $Y$ cannot be flatly smoothed on $F$. 
Actually more is true. $Y$ is not even the flat degeneration of an integral curve in $\mathbf{P}^{3}$ (and therefore in particular a degeneration of a nonsingular curve). Indeed it is easy to compute that $p_{a}(Y)=3$, degree $Y=5$. But from the Castelnuovo bound [1] the maximal possible arithmetic genus for an integral curve of degree 5 in $\mathbf{P}^{3}$ is 2 .

\section{REFERENCES}

1. P. Griffiths and J. Harris, Principles of algebraic geometry, Wiley, New York, 1978.

2. B. Saint-Donat, Projective models of $K-3$ surfaces, Amer. J. Math. 96 (1974), 602-639.

Department of Theoretical Mathematics, Weizmann Institute of Science, Rehovot 76100 , ISRAEL

Current address: Department of Mathematics, University of Florida, Gainesville, Florida 32611 